The final publication is available at Elsevier via http://dx.doi.org/10.1016/j.jtos.2016.04.001 (C) 2016. This manuscript version is made available under the CC-BY-NC-ND 4.0 license http://creativecommons.org/licenses/by-nc-nd/4.0/

Title:

Influence of conjunctival folds on calculated tear meniscus volume along the lower eyelid

Authors:

Stefan Bandlitz PhD,

Christine Purslow PhD,

Paul J Murphy PhD,

Heiko Pult PhD

School of Optometry and Vision Sciences, Cardiff University, Cardiff, Wales, United Kingdom (SB, CP, PJM, HP), and Cologne School of Optometry, Cologne, Germany (SB), and Dr. Heiko Pult -Optometry and Vision Research, Weinheim, Germany (HP), and University of Waterloo, School of Optometry and Vision Science, Waterloo, Canada (PJM)

\title{
Tables: 3
}

Figures: 6

Word count: 2793

\section{Corresponding Author}

Stefan Bandlitz

Höhere Fachschule für Augenoptik Köln (Cologne School of Optometry)

Bayenthalgürtel 6-8, D-50968 Köln, Germany

e-mail: bandlitz@hfak.de; Telephone: 0049-221-348080 
Purpose: When calculating tear meniscus volume (TMV), tear meniscus height $(\mathrm{TMH})$, radius (TMR) and cross-sectional area (TMA) are mostly measured at the centre of the lower lid margin. Lid-parallel conjunctival folds (LIPCOF) are known to influence the tear meniscus regularity. The aim of this study was to analyse the influence of LIPCOF on TMA measured by optical coherence tomography (OCT) and consequently, the calculated tear meniscus volume (TMV).

Methods: Using OCT (Cirrus-HD; Carl Zeiss Meditec, Jena, Germany), TMH, TMR and TMA of 42 subjects (13M, 29F; mean age $27.3 \mathrm{SD} \pm 8.4$ years) were measured directly below the pupil centre, plus at temporal and nasal locations perpendicularly below the limbus, where LIPCOF was also evaluated and graded. TMV for the different locations was calculated. Correlations between LIPCOF and the tear meniscus parameters were analysed using the Spearman Rank-Order coefficients. Differences between tear meniscus parameters at the different locations were evaluated by the paired t-test.

Results: Central TMV $\left(5.30 \pm 1.42 \times 10^{-2} \mu \mathrm{l} / \mathrm{mm}\right)$ was significantly positively correlated to LIPCOFsum $(2.4 \pm 1.2)(r=0.422 ; p<0.05)$. The calculated temporal TMV was greater by $0.53 \times 10^{-2} \mu \mathrm{l} / \mathrm{mm}$ compared to the central TMV $(p=0.037)$, while there was no significant difference in tear volume between the other locations. 
Conclusions: Using OCT it was possible to investigate the influence of LIPCOFs on $\mathrm{TMH}, \mathrm{TMR}$, and for the first time on TMA, at central and paracentral positions along the lower lid margin. The presence of LICPOFs results in an irregularity of tear meniscus with a difference in the amount of predicted tear volume while measuring $\mathrm{TMH}$ or TMR at the different locations.

Key words: tear meniscus, optical coherence tomography, LIPCOF, conjunctival folds, tear volume. 


\section{INTRODUCTION}

The tear fluid on the eye is present in three sections: at the exposed area between the lids covering the cornea and sclera, in the tear menisci at the lid margins, and in the conjunctival sacs of the upper and lower lid.[1] The tear menisci along the superior and inferior lid margins represent $75 \%$ to $90 \%$ of the tear film volume at the ocular surface,[2] although a lower estimate of $27 \%$ has been made.[1] The shape of the lower central meniscus is described to be roughly wedge-shaped in sagittal section, with a concave anterior surface, and posterior and peripheral surfaces that bathe and moisten the hydrophilic mucosae of the cornea and bulbar conjunctiva or palpebral conjunctiva.[3] However, the cross-sectional profile of the meniscus is likely to have a more complex shape,[3] with a parabolic anterior profile[4] and a posterior surface that is influenced by the shape of the underlying conjunctiva at the paracentral lid locations.[5]

At the central lid location, the evaluation of tear meniscus parameters is regarded as an indicator of tear film volume.[6, 7] The tear meniscus can be characterized by tear meniscus height $(\mathrm{TMH})$, tear meniscus radius (TMR) or cross-sectional tear meniscus area (TMA), and these have been shown to be significantly correlated to one another at the central tear meniscus.[8-11] For paracentral positions along the lower eyelid, however, the relationship between meniscus height, radius and crosssectional area has not yet been published.

The volume of the tear meniscus (TMV) has traditionally been calculated from $\mathrm{TMH}$, TMR or TMA of the central lower tear meniscus multiplied by the length of the lid margin. $[12,13]$ Since the meniscus is spread along the eyelid margins, variations in the measured meniscus parameters along the lid are likely to influence the 
calculation of the lower lid tear meniscus volume. Lid parallel conjunctival folds (LIPCOF) are folds in the inferotemporal and inferonasal quadrant of the bulbar conjunctiva, parallel to the lower lid margin. LIPCOF can be observed with the slitlamp or by optical coherence tomography (OCT), and they have been found to correlate with dry eye symptoms.[5, 14-19] Like conjunctivochalasis, LIPCOFs are assumed to alter the measurement of the tear meniscus area.[16, 18, 20, 21]

Using a portable digital meniscometer (PDM) it was shown that an increase in LIPCOF grade is associated with a higher $\mathrm{TMH}$ and a larger TMR at the nasal and temporal locations of the tear meniscus.[22] Furthermore, it was suggested that LIPCOF also impacts the central TMH evaluation, and that the presence of LIPCOF may cause the central $\mathrm{TMH}$ measurement to overestimate the actual central tear meniscus volume.[23] However, TMH and TMR measurements are limited to one dimension and describe only the anterior surface of the tear meniscus and do not account for the posterior section of the meniscus, so the volume of the LIPCOF is likely to influence the cross-sectional tear meniscus area (TMA).

Consequently, the aims of this study were: (i) to investigate the influence of LIPCOFs on $\mathrm{TMH}, \mathrm{TMR}$ and on TMA, measured by optical coherence tomography (OCT) at the central and paracentral position of the lower lid and (ii) to analyse the influence of LIPCOF on the calculated tear meniscus volume at the different locations. 


\section{MATERIALS AND METHODS}

\section{Subjects}

Forty-two subjects (male $=13$, female $=29$ ) were randomly selected from the staff and students of the Höhere Fachschule für Augenoptik Köln (Cologne School of Optometry), Cologne, Germany. The mean age of the subjects was $27.3 \pm 8.4$ (SD) years (range, 20 to 67 years). Subjects were excluded if they were pregnant or breast-feeding; had a current or previous condition known to affect the ocular surface or tear film; had a history of previous ocular surgery, including refractive surgery, eyelid tattooing, eyelid surgery, or corneal surgery; had any previous ocular trauma; were diabetic; were taking medication known to affect the ocular surface and/or tear film; and/or had worn contact lenses during the preceding two weeks prior to the study.

All subjects gave written informed consent before participating in the study. All procedures obtained the approval of the Cardiff School of Optometry and Vision Sciences Human Ethics Committee and were conducted in accordance with the requirements of the Declaration of Helsinki.

\section{Instrumentation and procedures}

OCT images of the lower tear meniscus were obtained during a single session using Cirrus HD-OCT (Carl Zeiss Meditec, Jena, Germany). This instrument uses spectral domain OCT (SD-OCT), with a wavelength of 840nm to achieve an axial resolution of $5 \mu \mathrm{m}$. The cross-sectional images of the tear meniscus in this study were taken using five vertically-oriented raster lines. In this mode, five parallel vertical lines of $3 \mathrm{~mm}$ length and a line distance of $0.25 \mathrm{~mm}$ were scanned; each line was composed of 
4096 A-scans. Tear meniscus scans were performed directly below the pupil centre, plus temporally and nasally tangential to the limbus (Figure 1).

OCT images were taken of the lower tear meniscus of the right eye in primary gaze, in a randomized order of the three locations by a single observer. To minimise diurnal and inter-blink variation, all measurements were taken in the morning between 10 and 12 o'clock and 3 to 4 seconds after a normal blink.

The OCT images were stored as jpeg files and image distortions were corrected as described previously by Bandlitz et al., 2014.[24] Using ImageJ 1.48 software (http://rsbweb.nih.gov/ij) on the OCT images, tear meniscus height (TMH) was measured as the distance of the intersection of the meniscus with the cornea/sclera and with the eyelid (Figure $2 \mathrm{~A}$ ). Tear meniscus radius (TMR) was calculated by applying a three-point circle fit technique (Figure 2 B). Tear meniscus area (TMA) was analyzed by the segmented-line function in ImageJ, where only the area of tear meniscus, but not the area of LIPCOF tissue, was marked (Figure $2 \mathrm{C}$ ).

Lid-parallel conjunctival folds were evaluated clinically without fluorescein using a slitlamp microscope (BQ900, Haag-Streit, Koeniz, Switzerland) using 25x magnification (Figure 3). The LIPCOF evaluation was performed in the area tangential to the temporal and nasal limbus, on the bulbar conjunctiva above the lower lid, at the same location where TMH, TMR and TMA were measured. LIPCOF grade was classified using an optimized grading scale (Table 1).[15, 25] LIPCOFsum was based on the sum of nasal and temporal LIPCOF scores. Care was taken to differentiate LIPCOF from micro-folds. This was done by evaluation of the fold thickness; the thickness of 
a single LIPCOF is approximately $0.08 \mathrm{~mm}$, while that of a micro-fold is $0.01 \mathrm{~mm}$.[23, $26,27]$

The study was conducted in a room with controlled temperature (20 to $23^{\circ} \mathrm{C}$ ) and humidity (44 to 53\%). Analysis of OCT tear meniscus parameters was masked against LIPCOF grading.

\section{Lower tear meniscus volume calculation}

As suggested by Palakuru et al.[6], the volume of the lower tear meniscus can be calculated from the cross-sectional TMA measured in the center of the eyelid multiplied by an average lid length of $25 \mathrm{~mm}$. Since the lower lid is curved along its length, a multiplication factor of 1.294 was suggested by Tiffany et al.[28]. However, in this calculation it is assumed that TMA is equally distributed along the lid. To account for variation in TMA and the influence of LIPCOFs on the tear volume along the lid, the volume that is present at the three different locations was calculated. According to Bitton et al.[29], it was assumed that TMA is similar across an eyelid length area of $1 \mathrm{~mm}$ at the location of the OCT cross-sectional scan and that in this small area the curvature of the lid is negligible. In consequence the following formula was used to calculate the tear volume at the temporal, central and nasal area:

$\mathrm{TMV}[\mu \mathrm{l}]=\mathrm{TMA}\left[\mathrm{mm}^{2}\right] \times 1 \mathrm{~mm}$ of lower lid length 


\section{Statistical methods}

Data were tested for normality using the Shapiro-Wilk test and appropriate statistical tests were applied. Correlations were calculated with Pearson correlation (or Spearman rank in non-parametric data). The differences between the locations along the lower lid were calculated with the paired t-test.

\section{RESULTS}

\section{Differences of tear meniscus parameter at the different locations}

Mean values and standard deviations for the tear meniscus parameters at the different locations are summarised in Table 2. Compared to TMH measured in the central location, $\mathrm{TMH}$ at the temporal location was $0.088 \pm 0.102 \mathrm{~mm}$ higher and at the nasal locations was $0.044 \pm 0.081 \mathrm{~mm}$ higher $(p<0.001)$. Temporal TMH was also found to be $0.044 \pm 0.130 \mathrm{~mm}$ higher than nasal TMH $(p<0.05)$. Compared to TMR measured in the central location, TMR at the temporal location was $0.063 \pm 0.061 \mathrm{~mm}$ larger $(p=0.009)$. However, no significant differences were found between nasal TMR and central TMR $(p=0.073)$ or temporal TMR $(p=0.804)$. Compared to TMA measured in the central location, TMA at the temporal location was $0.0053 \pm 0.0159 \mathrm{~mm}^{2}$ greater $(p=0.037)$, while there was no significant difference between nasal TMA and central TMA $(p=0.110)$ or temporal TMA $(p=0.628)$. Consequently, the calculated temporal TMV was increased by $0.53 \times 10^{-2} \mu \mathrm{l} / \mathrm{mm}$ compared to the central TMV $(p<0.05)$, while there was no statistically difference in tear volume between the other locations.

\section{Correlations between LIPCOFs and tear meniscus parameters}


Temporal LIPCOF grade $(1.4 \pm 0.9)$ was significantly positively correlated to all temporal tear meniscus parameters (temporal TMH:r $=0.547 ; p<0.001$; temporal TMR: $r=0.520 ; p<0.001 ;$ temporal TMA:r=0.368; $p=0.02$ ). Nasal LIPCOF grade $(0.6 \pm 0.8)$ was correlated to nasal TMR $(r=0.369 ; p=0.018)$, but not to nasal TMH $(p=0.095)$, nor to nasal TMA $(p=0.278)$. LIPCOFsum $(2.4 \pm 1.2)$ was significantly correlated to central TMH $(r=0.393 ; p=0.01)$, central TMR $(r=0.350 ; p=0.02)$ and central TMA $(r=0.422 ; p<0.05)$.

\section{Calculated tear meniscus volume at the different locations}

Significant correlations were observed between the centrally calculated TMV $\left(5.30 \pm 1.42 \times 10^{-2} \mu \mathrm{l} / \mathrm{mm}\right)$ and the centrally measured TMH $(r=0.968 ; p<0.001)$ and TMR $(r=0.837 ; p<0.001) \quad($ Figure $4-5)$. Temporal calculated TMV $\left(5.83 \pm 2.13 \times 10^{-2} \mu \mathrm{l} / \mathrm{mm}\right)$ was correlated to temporal TMH $(r=0.796 ; p<0.001)$ and temporal TMR $(r=0.743 ; p<0.001)$, while nasal calculated TMV $\left(5.45 \pm 1.94 \times 10^{-2} \mu \mathrm{l} / \mathrm{mm}\right)$ was correlated to nasal TMH $(r=0.897 ; p<0.001)$ and nasal TMR $(r=0.830 ; p<0.001)$ (Figure 4-5).

To account for any difference in the amount of predicted tear volume while measuring an equal TMH or TMR at the different locations, a linear regression analysis for each location was calculated and formula given in the graph (Figures 4 and 5). In order to allow the clinician to compare the amount of calculated TMV typical values of 0.1 to 1.0 for $\mathrm{TMH}$ and TMR were used as an independent to calculate the dependent TMV for each location (Table 3).

\section{DISCUSSION}


The aim of the study was to use optical coherence tomography to investigate the influence of LIPCOFs on TMH, TMR, and for the first time on TMA (and therefore TMV), at the central and para-central positions along the lower lid margin. For the central $\mathrm{TMH}$, an increasing height was correlated to LIPCOFsum. This is in concordance with a recently published study in which a slit-lamp with image analysis software was used to measure the central tear meniscus.[23] On the high-resolution, cross-sectional OCT images used in the present study, it was furthermore possible to analyse TMR and TMA, which for the central lid position were also correlated to LIPCOFsum. Temporal LIPCOF seemed to impact temporal TMH, TMR and TMA, while nasal LIPCOF only appear to impact nasal TMR. This difference between temporal and nasal is likely to be caused by the unequal LIPCOF grades at these locations. However, it has to be mentioned that LIPCOF grades in this study were small due to that the subjects were normals and had no dry eyes. It was suggested that for LIPCOF grades greater than or equal to 2, an irregularity of TMH and TMR along the lower lid could be expected.[22] Nemeth et al.[30] suggested that the sensitivity and specificity of LIPCOF grading for discriminating between normal and dry eyes were best with the cut-off between LIPCOF degrees 1 and 2, which supports other findings of LIPCOF being a good discriminator between normal and dry eye patients.[14, 22] From this it can be hypothesized that an irregularity in $\mathrm{TMH}$ and TMR in the central zone of the lower tear meniscus would be caused by LIPCOFs and therefore is an indicator for dry eye patients. Others reported a relationship between tear meniscus irregularity and dry eye symptoms before,[31-33] however in these studies the degree of LIPCOFs was not analysed.

$\mathrm{TMH}$ and TMR at the para-central location was higher or flatter, respectively, compared to the central location, which is in agreement with our previously published 
study.[22] Interestingly, tear meniscus area, and therefore tear meniscus volume, was not increased for the nasal location while it was for the temporal location. From this it might be hypothesized the nasal degree of LIPCOFs increase $T M H$ and TMR, but the effect is not sufficient to also influence TMA and consequently tear volume at this location. This is likely to be caused by the fact that the tissue of the conjunctival folds protrudes into the cross-sectional area of the meniscus fluid (Figure $2 \mathrm{C}$ ). While doing so, TMH and TMR is rising, but since the tear volume is only displaced by the folds it remains constant. On the other hand, for the temporal area with the higher LIPCOF degrees, LICPOFs also cause an increase in TMA and, therefore, tear volume. However, the increase in tear volume at the temporal location is different from the increase in tear volume that would be expected from measuring $\mathrm{TMH}$ or TMR at the central location or nasal location (Table 3). Consequently, for a constant TMH or TMR, the associated TMA value was different at each of the three locations (central, nasal, and temporal) (Figure 6).

For the temporal TMA, Gumus et al.[34] reported a significant increase after cauterization of conjunctivochalasis. This means that by reducing the amount of conjunctival tissue in the cross-sectional tear meniscus area, the tear volume at this location will increase. LIPCOF have been described as a sub-type of conjunctivochalasis that might represent a mild stage.[35] This hypothesis may be supported by the finding that the cross-sectional area of LIPCOF tissue appeared to be much smaller than that of conjunctivochalasis, and that even after conjunctivochalasis treatment the remaining tissue is still commonly much larger than LIPCOF.[23] However, with an increasing LIPCOF grade, an increase in crosssectional TMA was found for the temporal location. This seems to be conflicting with the findings of a reduced TMA in conjunctivochalasis. From this it may be 
hypothesised that a small amount of conjunctival tissue that protrudes into the meniscus results in an increase in volume, while a large amount of tissue results in a decrease of tear volume at this location. So it is likely that there is a turning point at which an increasing cross-sectional area of conjunctival tissue in the meniscus induces a decreasing tear volume.

Furthermore, it might be concluded that even if conjunctivochalasis and LIPCOF both interfere with the meniscus, they have different impacts on the distribution of tear fluid along the lower eyelid.

However, in the existing studies on LIPCOF and conjunctivochalasis, tear meniscus parameters were observed in the lid area from limbus to limbus, which represents approximately $12 \mathrm{~mm}$ of the total lid length of about $25 \mathrm{~mm}$. Consequently the impact of conjunctival folds on the total tear meniscus, and therefore on the overall tear meniscus volume, remains unknown. There is only one report in the literature in which $\mathrm{TMH}$ measured in the nasal and temporal areas $3 \mathrm{~mm}$ from the nasal and temporal canthi was found to be lower than central $\mathrm{TMH}$, but it was not noted whether conjunctival folds were present in this study.[13]

In summary, using OCT tear meniscus parameters, it was possible to investigate the influence of LIPCOFs on TMH, TMR and for the first time on TMA at the central and paracentral position of the lower lid. The presence of LIPCOFs results in an irregularity of the tear meniscus along the lid length, and it is also associated with a variation in the relationship between tear volume and tear meniscus height or radius. 


\section{REFERENCES}

[1] Gaffney EA, Tiffany JM, Yokoi N, Bron AJ. A mass and solute balance model for tear volume and osmolarity in the normal and the dry eye. Prog Retin Eye Res. 2010;29:5978.

[2] Holly FJ. Physical chemistry of the normal and disordered tear film. Trans Ophthalmol Soc U K. 1985;104 ( Pt 4):374-80.

[3] Bron AJ, Yokoi N, Gaffney EA, Tiffany JM. A solute gradient in the tear meniscus. I. A hypothesis to explain Marx's line. Ocul Surf. 2011;9:70-91.

[4] Bandlitz S, Purslow C, Murphy PJ, Pult H. Evaluation of lower tear meniscus shape with OCT. Acta Ophthalmologica2012. p. 0. doi: 10.1111/j.755-3768.2012.4641.x.

[5] Veres A, Tapaszto B, Kosina-Hagyo K, Somfai GM, Nemeth J. Imaging lid-parallel conjunctival folds with OCT and comparing its grading with the slit lamp classification in dry eye patients and normal subjects. Invest Ophthalmol Vis Sci. 2011;52:2945-51.

[6] Palakuru JR, Wang J, Aquavella JV. Effect of blinking on tear dynamics. Invest Ophthalmol Vis Sci. 2007;48:3032-7.

[7] Yokoi N, Bron AJ, Tiffany JM, Maruyama K, Komuro A, Kinoshita S. Relationship between tear volume and tear meniscus curvature. Arch Ophthalmol. 2004;122:1265-9.

[8] Golding TR, Bruce AS, Mainstone JC. Relationship between tear-meniscus parameters and tear-film breakup. Cornea. 1997;16:649-61.

[9] Mainstone JC, Bruce AS, Golding TR. Tear meniscus measurement in the diagnosis of dry eye. Curr Eye Res. 1996;15:653-61.

[10] Oguz H, Yokoi N, Kinoshita S. The height and radius of the tear meniscus and methods for examining these parameters. Cornea. 2000;19:497-500.

[11] Shen M, Li J, Wang J, Ma H, Cai C, Tao A, et al. Upper and lower tear menisci in the diagnosis of dry eye. Invest Ophthalmol Vis Sci. 2009;50:2722-6. 
[12] Wang J, Simmons P, Aquavella J, Vehige J, Palakuru J, Chung S, et al. Dynamic distribution of artificial tears on the ocular surface. Arch Ophthalmol. 2008;126:619-25. [13] Jones LW, Rahman S, Leech R, Simpson T, Fonn D. Determination of Inferior Tear Meniscus Height and Inferior Tear Meniscus Volume Using Optical Coherence Tomography Invest Ophthalmol Vis Sci 2004. p. E-Abstract 144.

[14] Pult H, Purslow C, Murphy PJ. The relationship between clinical signs and dry eye symptoms. Eye (Lond). 2011;25:502-10.

[15] Pult H, Purslow C, Berry M, Murphy PJ. Clinical tests for successful contact lens wear: relationship and predictive potential. Optom Vis Sci. 2008;85:E924-9.

[16] Tapaszto B, Veres A, Kosina-Hagyo K, Somfai GM, Nemeth J. OCT Imaging of lidparallel conjunctival folds in soft contact lens wearers. Optom Vis Sci. 2011;88:1206-13. [17] Hoh H, Schirra F, Kienecker C, Ruprecht KW. [Lid-parallel conjunctival folds are a sure diagnostic sign of dry eye]. Ophthalmologe. 1995;92:802-8.

[18] Efron N, Jones L, Bron AJ, Knop E, Arita R, Barabino S, et al. The TFOS International Workshop on Contact Lens Discomfort: Report of the Contact Lens Interactions With the Ocular Surface and Adnexa Subcommittee. Invest Ophthalmol Vis Sci. 2013;54:TFOS98TFOS122.

[19] Craig JP, Willcox MD, Argueso P, Maissa C, Stahl U, Tomlinson A, et al. The TFOS International Workshop on Contact Lens Discomfort: Report of the Contact Lens Interactions With the Tear Film Subcommittee. Invest Ophthalmol Vis Sci. 2013;54:TFOS123-56.

[20] Gumus K, Crockett CH, Pflugfelder SC. Anterior segment optical coherence tomography: a diagnostic instrument for conjunctivochalasis. Am J Ophthalmol. 2010;150:798-806. 
[21] Huang Y, Sheha H, Tseng SC. Conjunctivochalasis interferes with tear flow from fornix to tear meniscus. Ophthalmology. 2013;120:1681-7.

[22] Bandlitz S, Purslow C, Murphy PJ, Pult H. The Relationship between Tear Meniscus Regularity and Conjunctival Folds. Optom Vis Sci. 2014;91:1037-44.

[23] Pult H, Riede-Pult BH. Impact of conjunctival folds on central tear meniscus height. Invest Ophthalmol Vis Sci. 2015;56:1459-66.

[24] Bandlitz S, Purslow C, Murphy PJ, Pult H. Comparison of a new portable digital meniscometer and optical coherence tomography in tear meniscus radius measurement. Acta Ophthalmol. 2014;92:e112-8.

[25] Berry M, Pult H, Purslow C, Murphy PJ. Mucins and ocular signs in symptomatic and asymptomatic contact lens wear. Optom Vis Sci. 2008;85:E930-8.

[26] Markoulli M, Carnt N, Jalbert I, Keay L, Naduvilath T, E. P. Resolution and Clinical Characteristics of Conjunctival "Flaps". Invest Ophthalmol Vis Sci. 2007;48:ARVO EAbstract:5391.

[27] Thota S, Perrigin J, Miller W, Leach N, Bergmanson J, A. B. Conjunctival Flaps in Silicone Hydrogel Lens Wearers. Invest Ophthalmol Vis Sci. 2006;47:ARVO E-Abstract: 82.

[28] Tiffany JM, Todd BS, Baker MR. Computer-assisted calculation of exposed area of the human eye. Adv Exp Med Biol. 1998;438:433-9.

[29] Bitton E, Keech A, Simpson T, Jones L. Variability of the analysis of the tear meniscus height by optical coherence tomography. Optom Vis Sci. 2007;84:903-8.

[30] Nemeth J, Fodor E, Lang Z, Kosina-Hagyo K, Berta A, Komar T, et al. Lid-parallel conjunctival folds (LIPCOF) and dry eye: a multicentre study. $\mathrm{Br} \mathrm{J}$ Ophthalmol. 2012;96:1380-5. 
[31] Guillon JP. Non-invasive Tearscope Plus routine for contact lens fitting. Cont Lens Anterior Eye. 1998;21:45-8.

[32] Holly FJ, Lemp MA. Tear physiology and dry eyes. Surv Ophthalmol. 1977;22:69-87. [33] Taylor HR. Studies on the tear film in climatic droplet keratopathy and pterygium. Arch Ophthalmol. 1980;98:86-8.

[34] Gumus K, Pflugfelder SC. Increasing prevalence and severity of conjunctivochalasis with aging detected by anterior segment optical coherence tomography. Am J Ophthalmol. 2013;155:238-42 e2.

[35] Schirra F, Hoh H, Kienecker C, Ruprecht KW. Using LIPCOF (lid-parallel conjunctival fold) for assessing the degree of dry eye, it is essential to observe the exact position of that specific fold. Adv Exp Med Biol. 1998;438:853-8. 


\section{Tables}

Table 1. Optimized Grading Scale of LIPCOF.[14, 25]

Table 2. Mean values and standard deviations for the tear meniscus parameters and LIPCOF grades at the different locations along the lower eyelid.

Table 3. Predicted tear volume for typical values of $T M H$ and TMR at the different location along the lower eyelid.

\section{Figures}

Figure 1. Anterior segment 5 lines raster of the Cirrus HD-OCT, showing the observer's view and the alignment targets at the three locations along the lower lid.

Figure 2A. Tear meniscus height $(\mathrm{TMH})$ measured on the optical coherence tomography (OCT) image using the straight-line tool in ImageJ.

Figure 2B. Tear meniscus radius (TMR) measured on the optical coherence tomography (OCT) image using the 3-point line-fit technique in ImageJ.

Figure 2C. Tear meniscus cross-sectional area (TMA) measured on the optical coherence tomography (OCT) image using the segmented-line tool in ImageJ.

Figure 3. Slit lamp image of LIPCOF grade 3 at the temporal position. 
Figure 4. Linear regression to describe the relationship between measured TMH and tear volume at the different locations.

Figure 5. Linear regression to describe the relationship between measured TMR and tear volume at the different locations.

Figure 6. Examples of tear meniscus cross-sectional areas (TMA) and LIPCOF grades at the different locations. 